\title{
Factores de riesgo para la presentación de conductas no deseadas en equinos de deporte en Chile
}

\author{
Risk factors for the presentation of undesired behaviours in sport horses in Chile \\ D Navarrete ${ }^{\mathrm{a}}$, C Hamilton-West ${ }^{\mathrm{b}}$, N Stephens ${ }^{\mathrm{c}}, \mathbf{C}$ Weber $^{\mathrm{d}}$, T Tadich ${ }^{\mathrm{a}}$ \\ ${ }^{a}$ Departamento de Fomento de la Producción Animal, Facultad de Ciencias Veterinarias y Pecuarias, \\ Universidad de Chile, Santiago, Chile. \\ bepartamento de Medicina Preventiva Animal, Facultad de Ciencias Veterinarias y Pecuarias, \\ Universidad de Chile, Santiago, Chile. \\ ${ }^{\mathrm{c} E s c u e l a}$ de Ciencias Veterinarias, Universidad Viña del Mar, Viña del Mar, Chile. \\ ${ }^{\mathrm{d}}$ Facultad de Ciencias Veterinarias, Universidad Austral de Chile, Valdivia, Chile.
}

\begin{abstract}
SUMMARY
Behaviour alterations are considered as cause and symptom of poor animal welfare. Generally they have been associated to suboptimal husbandry practices and intrinsic factors of horses. The aim of this study was to indentify risk factors for the presentation of behaviour alterations in sport horses. The information of 1,529 equines was compiled from three databases, the individuals were classified in groups according to the type of behaviour in "locomotor" and "oral". Environmental and intrinsic conditions were evaluated as possible risk factors for the presentation of oral behavioural alterations through a multiple logistic regression model; a significance level of $\mathrm{P}<0.05$ was considered. A total of 188 individuals with behavioural alterations were registered, and thirteen of them presented more than one alteration. A total of 90 locomotor and 111 oral undesired behaviours were registered; the most frequent undesired behaviours were box-walking and crib-biting/wind-sucking for each group respectively. In the case of locomotor behaviours no risk factors statistically significant were found, however a forage percentage $\geq 60 \%$ in the ration was identified as a protective factor, as was belonging to the Chilean breed. On the other hand, for the oral behaviours, a forage percentage $\geq 60 \%$ was identified as a statistically significant risk factor, and the Thoroughbred breed was identified as protective factor in the logistic regression. Some management factors and intrinsic characteristics of the animal may increase or decrease the presentation of these behaviours in sport horses, these factors should be considered when designing an equine production system in order to maintain their welfare.
\end{abstract}

Key words: equine, behaviour, animal welfare, risk factors.

\section{RESUMEN}

Las alteraciones conductuales son consideradas causa y síntoma de un problema de bienestar animal. Generalmente se han asociado a condiciones de estabulación o manejo subóptimas, y a factores intrínsecos del animal. El objetivo del estudio fue identificar factores de riesgo para la presentación de alteraciones conductuales en equinos de deporte. Se recopiló información de 1.529 equinos de tres bases de datos y se clasificaron en grupos según el tipo de conducta no deseada: locomotor y oral. Las condiciones ambientales e intrínsecas fueron evaluadas como posibles factores de riesgo para la presentación de alteraciones conductuales mediante un modelo de regresión logística multivariado, estableciéndose un nivel de significancia de $\mathrm{P}<0,05$. Se registraron en total 188 individuos con alteraciones conductuales, de los cuales trece presentaron más de una. Se presentaron 90 conductas locomotoras y 111 orales, siendo la caminata estereotipada y la aerofagia las más frecuentes en cada grupo. Para el caso de las conductas de tipo locomotor no se encontraron factores de riesgo estadísticamente significativos. Sin embargo, se identificó como factor protectivo un porcentaje de forraje $\geq 60 \%$ en la ración y la raza Caballo Chileno. Por otro lado, para las conductas orales, un porcentaje de forraje $\geq 60 \%$ fue identificado como un factor de riesgo estadísticamente significativo, mientras que pertenecer a la raza FSC se identificó como factor protectivo. Ciertos factores de manejo y características intrínsecas del animal pueden aumentar o disminuir la presentación de estas conductas en los equinos de deporte, siendo factores importantes de considerar al momento de diseñar un sistema de manejo de equinos cuidando su bienestar.

Palabras clave: equinos, conducta, bienestar animal, factor de riesgo.

\section{INTRODUCCIÓN}

Los equinos son utilizados en diversas actividades como deporte, trabajo y producción de carne, para lo cual generalmente se mantienen en sistemas de estabulación. Este

Aceptado: 26.06.2014.

* Santa Rosa 11735, La Pintana, Santiago, Chile; tamaratadich@ u.uchile.cl sistema reduce las oportunidades de realizar actividades presentes en su repertorio conductual normal (Hothersall y Nicol 2009). Esta restricción del repertorio conductual normal se ha relacionado con el desarrollo de conductas no deseadas. La mayoría de las conductas no deseadas en los equinos resultan de dos cambios fundamentales que caracterizan su vida en estado salvaje, los que se encuentran ausentes en cautiverio. Por una parte, la estructura social se ve alterada, ya que el equino es un animal gregario que 
forma parte de un harem o de bandas de machos juveniles, y que raramente se mantiene solitario por opción propia, lo cual generalmente no ocurre dentro del manejo en cautiverio. Por otra parte, la disponibilidad ilimitada de tiempo para forrajear se ve notablemente restringida en cautiverio (Boyd 1986, Houpt 1986).

Dentro de las conductas no deseadas se encuentran las estereotipias, como el balanceo estereotipado, conocido popularmente como mal o baile del oso; la aerofagia con o sin fijación; y la caminata estereotipada (Tadich y Araya 2010). Las estereotipias se definen como patrones de comportamiento repetitivos e invariables sin un objetivo o función obvia (Mason 1991), las que se sugiere podrían desarrollarse como conductas adaptativas para aminorar los efectos negativos de un ambiente subóptimo presente o pasado en la vida del animal (Mason 1991, Hothersall y Casey 2012). Además, existen otros tipos de conductas no deseadas como las redirigidas o conductas dirigidas a un objeto diferente a la fuente de estímulo, por ejemplo, la lignofagia; las desplazadas, que son una respuesta inapropiada frente a una situación en particular, como el manoteo, y las aprendidas, como ciertas conductas agresivas (McGreevy 2004).

La importancia de identificar los factores de riesgo para la presentación de estas conductas y lograr prevenir su desarrollo radica en que en Chile alrededor del $50 \%$ de los criaderos de equinos de la raza Caballo Chileno tiene uno o más ejemplares con estas conductas (Muñoz y col 2009). Además, existiría una prevalencia de estereotipias de $6,32 \%$ y un $5,52 \%$ para otras conductas anormales en equinos (FSC) en Chile (Tadich y col 2013). Sumado a esto, se ha descrito que cuando la conducta estereotipada se establece en el repertorio del animal, esta persiste sin importar los intentos por mejorar las deficiencias que las causan (McGreevy 2004), por lo demás el castigo o la prevención con medios físicos generalmente es detrimental para el bienestar del equino y no soluciona los factores causales presentes en el ambiente (Mills y col 2005, Tadich y Araya 2010).

\section{MATERIAL Y MÉTODOS}

\section{OBTENCIÓN DE DATOS}

Se utilizó información de 1.529 equinos, de diferente edad, sexo, raza y actividad, proveniente de tres bases de datos. La primera fue generada en el Club Hípico de Santiago y el Hipódromo Chile, con información de equinos FSC $(n=742)$ (Weber 2010). La segunda base de datos $(n=554)$ corresponde a equinos de salto y de carrera, cuyos datos se obtuvieron en el Club de Polo y Equitación San Cristóbal y el Santiago Paperchase Club, ubicados en la Región Metropolitana, además de la Escuela de Caballería Blindada del Ejército y el Valparaíso Sporting Club, en la Región de Valparaíso (Stephens 2011), y por último datos de una muestra de equinos Caballo Chileno utilizados para rodeo $(\mathrm{n}=232)$ correspondiente a información de 23 centros de crianza ubicados en las Regiones del Maule, de los Ríos, de los Lagos y de Aysén (Tadich 2012). Los 1.529 equinos registrados corresponden a un porcentaje de entre el $30 \%$ y $50 \%$ de los equinos de cada centro ecuestre. De las bases de datos originales aquellos individuos que no presentaban información, para una o más de las variables incluidas en el presente estudio, fueron excluidos a modo de obtener una base de datos homogénea, en el caso de la base de datos de Caballo Chileno se excluyeron los potrillos menores de un año y las yeguas que no eran ocupadas para función de deporte.

A partir de la información disponible y utilizando el programa Microsoft Office Excel 2007 (Microsoft Corporation) se generó una nueva base de datos y se establecieron las variables a analizar. Las conductas no deseadas fueron definidas y clasificadas como estereotipadas y otras conductas no deseadas y de acuerdo con su forma de presentación en orales y locomotoras (cuadro 1).

Se realizó un estudio del tipo casos y controles. En cada uno de estos grupos de conductas no deseadas, los equinos fueron clasificados como "caso" cuando presentaron alguna alteración conductual, si un equino presentaba más de una conducta cada conducta se consideró como caso ( $\mathrm{n}=201)$ y se consideraron como "control" cuando no existieron dichas alteraciones. Las variables asociadas al individuo y factores ambientales registrados se presentan en el cuadro 3.

Las variables raza y función se analizaron como una sola, ya que todos los individuos de raza Caballo Chileno tenían como función el rodeo, aquellos FSC función de carrera y todos aquellos Mestizos cumplían la función de salto, por lo que analizarlas como variables independientes generaría confusión.

\section{ANÁLISIS ESTADÍSTICO}

La asociación entre los factores individuales, ambientales y de manejo y la presentación de conductas no deseadas fue evaluada mediante regresión logística. Para esto se establecieron dos modelos, mediante un procedimiento paso a paso y eliminación hacia atrás, con un valor de $\mathrm{p}$ liberal de 0,20. En el primero se consideró como variable dependiente la presencia de conductas orales y en el segundo modelo se consideró como variable dependiente a la presencia de conductas locomotoras.

Se evaluó la presencia de interacciones y de factores de confusión, según la metodología propuesta por Hosmer and Lemeshow (2000), Las variables que no resultaron significativas, pero ejercieron ciertos cambios en los OR y en los valores de $\mathrm{p}$ de otras variables, fueron mantenidas en el modelo final por su efecto de confusión.

Se utilizó el programa Infostat versión 2012e (UNC, Córdoba, Argentina), estableciéndose un nivel de significancia de $\mathrm{P}<0,05$. De esta forma se estimó el valor odds 
Cuadro 1. Etograma de las conductas observadas y su clasificación en estereotipadas u otras conductas no deseadas y de acuerdo con su forma de presentación en oral o locomotora.

Ethogram of the behaviours observed in the present study. Each behaviour is classified as stereotype or other undesired behaviour and according to the form of presentation in oral or locomotor.

\begin{tabular}{|c|c|c|c|}
\hline \multicolumn{2}{|r|}{ Conducta } & Oral/Locomotor & Descripción \\
\hline \multirow{3}{*}{ 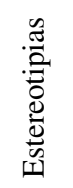 } & Aerofagia & Oral & Ingreso de aire hacia el esófago con o sin apoyo de los incisivos. \\
\hline & Balanceo estereotipado & Locomotor & Movimiento de balanceo lateral del tren anterior. \\
\hline & Caminata estereotipada & Locomotor & Caminar repetidamente dentro de la pesebrera \\
\hline \multirow{5}{*}{ 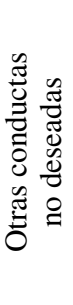 } & Lignofagia & Oral & Ingestión de madera. \\
\hline & Comer cama & Oral & Ingestión del material de cama. \\
\hline & Otras conductas orales & Oral & Lamer o morder objetos sin fin nutricional. \\
\hline & Cabeceo & Locomotor & Movimientos verticales repetidos de la cabeza. \\
\hline & Agresividad & Locomotor & Amenazas de o realización de mordidas o patadas dirigidas a humanos. \\
\hline
\end{tabular}

ratio $(\mathrm{OR})$ de las variables estudiadas, como un indicador de la asociación entre las variables.

\section{RESULTADOS}

En relación con la frecuencia de presentación de conductas no deseadas de origen oral y locomotora; prácticas de manejo; y características individuales en equinos de deporte, del total de 1.529 ejemplares estudiados, un $12 \%$ (188 individuos) presentó conductas no deseadas de cualquier tipo, ya sea oral o locomotor. Se identificó un total de 201 conductas no deseadas, ya que trece de los equinos desarrollaron más de una conducta. De estas conductas, las de tipo oral tuvieron una frecuencia de presentación levemente superior (cuadro 2). La conducta no deseada que se presentó con mayor frecuencia fue la aerofagia con o sin fijación, seguida de lignofagia, balanceo y caminata estereotipada (cuadro 2).

En relación con el sexo y raza (función) la mayor proporción de ejemplares fueron hembras y de raza FSC (cuadro 3). En lo que se refiere a prácticas de manejo, cerca de la totalidad de los individuos estudiados disponía de viruta como material de cama; la mayor proporción de los individuos eran alimentados con dietas que incluían menos de un $60 \%$ de forraje en la ración, porcentaje establecido como punto de corte de acuerdo con las recomendaciones de consumo esperado para equinos adultos en trabajo moderado o intenso (NRC 1989). La frecuencia de alimentación preponderante fue de tres veces al día. Aproximadamente el $90 \%$ de los individuos poseía contacto visual entre sí durante el tiempo de estabulación (cuadro 3).

El modelo de regresión logística para las conductas de tipo oral, identificó asociaciones significativas para la cantidad de forraje $\geq 60 \%$ en la ración $(\mathrm{OR}=2,17$,
Cuadro 2. Resumen de las frecuencias absolutas (FA) y porcentaje de presentación de cada conducta y de su clasificación de acuerdo con orales y estereotipadas. Trece equinos presentaron más de una conducta $(n=1.529)$.

Summary of the absolute frequencies (FA) and percentage of presentation for each behaviour and according to their classification in oral and locomotor. Thirteen horses showed more than one behaviour $(\mathrm{n}=1,529)$.

\begin{tabular}{|c|c|c|c|}
\hline & Conducta & FA & $\%$ \\
\hline \multirow{6}{*}{$\frac{\tilde{\sigma}}{\tilde{\sigma}} \frac{0}{0}$} & Aerofagia & 50 & 3,3 \\
\hline & Coprofagia & 7 & 0,5 \\
\hline & Lignofagia & 45 & 3,0 \\
\hline & Comer cama & 6 & 0,4 \\
\hline & Otras conductas orales & 3 & 0,2 \\
\hline & Total conductas orales & 111 & 7,4 \\
\hline \multirow{7}{*}{$\begin{array}{l}\tilde{\Xi} \\
0 \\
0 \\
0 \\
0 \\
0 \\
0 \\
.\end{array}$} & Balanceo estereotipado & 28 & 2,0 \\
\hline & Patear la pesebrera & 9 & 0,6 \\
\hline & Manoteo & 4 & 0,3 \\
\hline & Caminata estereotipada & 31 & 2,0 \\
\hline & Cabeceo & 5 & 0,3 \\
\hline & Agresividad & 13 & 0,9 \\
\hline & Total conductas locomotoras & 90 & 6,1 \\
\hline
\end{tabular}

$\mathrm{P}=0,0001)$, y para la raza $\mathrm{FSC}(\mathrm{OR}=0,58, \mathrm{P}=0,036)$ comparadas con la raza Caballo Chileno de rodeo y Mestizos de salto, mientras que las otras variables no fueron significativas (cuadro 4).

Para los factores de riesgo para conductas de tipo locomotor, el modelo de regresión logística identificó una asociación significativa para el porcentaje de forraje $\geq 60 \%$ en la ración $(\mathrm{OR}=0,47$, y a la raza Caballo 
Chileno $(\mathrm{OR}=0,08)$ al ser comparada con caballos de salto, mientras que las otras variables no resultaron significativas (cuadro 4).

\section{DISCUSIÓN}

Si bien este estudio combina el uso de diferentes bases de datos, que fueron creadas en diferentes momentos y condiciones, logra reunir un importante número de individuos

Cuadro 3. Descripción y categorización de las variables del animal y ambientales registradas con sus respectivas frecuencias absolutas (FA) y porcentaje de presentación dentro de la población $(\mathrm{n}=1.529)$.

Description and categorization of the animal and environmental variables registered with their respective absolute frequency (FA) and percentage of presentation within the population $(\mathrm{n}=1,529)$.

\begin{tabular}{llrc}
\hline Variable & Descripción & FA & Porcentaje \\
\hline \multirow{3}{*}{ Raza } & Mestizo & 267 & 18 \\
& FSC & 1.030 & 67 \\
& Caballo Chileno & 232 & 15 \\
\hline \multirow{2}{*}{ Sexo } & Macho castrado & 512 & 33 \\
& Macho entero & 361 & 24 \\
& Hembra & 656 & 43 \\
\hline \multirow{2}{*}{ Forraje } & $<60 \%$ & 1.031 & 67 \\
& $\geq 60 \%$ & 498 & 33 \\
\hline \multirow{2}{*}{ Material cama } & Viruta & 1.339 & 88 \\
& Paja & 190 & 12 \\
\hline \multirow{2}{*}{$N^{o}$ raciones } & 2 diarias & 541 & 35 \\
& 3 diarias & 988 & 65 \\
\hline \multirow{2}{*}{ Contacto visual } & Ausente & 189 & 12 \\
& Presente & 1.340 & 88 \\
\hline
\end{tabular}

en los que se evalúa la presencia de alteraciones conductuales y se asocian a diferentes condiciones de manejo y características del animal. Asimismo, se debe considerar que la metodología seleccionada para el análisis de los datos, permite establecer asociaciones entre una variable respuesta dicotómica, en este caso la presencia de las conductas no deseadas, y una o más variables explicatorias. Esta metodología ha sido reconocida y utilizada ampliamente en diferentes áreas; por ejemplo epidemiología, investigaciones biomédicas, ecología, ciencias políticas, entre otros (Hosmer y Lemeshow 2000).

Se han realizado diversos estudios a modo de determinar la prevalencia de problemas conductuales en equinos, siendo gran parte ellos realizados sobre poblaciones de equinos FSC mantenidos con fines deportivos. La mayoría de estos estudios reportan solo las frecuencias de presentación de aquellas conductas clasificadas como estereotipadas (aerofagia, balanceo y caminata estereotipada). Es así como en el estudio de McGreevy (1997) se registró una prevalencia de estereotipias mayor a los porcentajes de positividad registrados en este estudio para estereotipias (7,3\%), llegando a un 10,8\% al evaluar aerofagia, balanceo y caminar estereotipado en equinos FSC en el Reino Unido, mientras que posteriormente Pells y McGreevy (1999) registraron un 11,7\% para estas mismas conductas en equinos FSC en Australia. Tadich y col (2013) para esta misma raza en Chile registraron prevalencias menores tanto para conductas estereotipadas $(6,32 \%)$ como para otras conductas no deseadas $(5,52 \%)$ que aquellas reportadas en el extranjero, pero similares a los porcentajes de positividad identificados en el presente estudio (7,3 y 6,2\% respectivamente). Para el caso de la raza Caballo Chileno se obtuvo una prevalencia de 11,1\% de conductas no deseadas, de las cuales $3,1 \%$ correspondieron a estereotipias y $8 \%$ a otras conductas no deseadas (Tadich y col 2012). Cifras similares a las obtenidas en el

Cuadro 4. Resultados de la regresión logística multivariada (Odds ratio (OR), intervalos de confianza (CI) y valor de P) para conductas no deseadas de tipo oral y locomotor.

Results of the multivariate logistic regression (Odds ratio (OR), confidence intervals (IC) and P value) for oral and locomotor undesired behaviours.

\begin{tabular}{lcccccc}
\hline & \multicolumn{7}{c}{ Regresión logística multivariada } \\
\cline { 2 - 7 } & \multicolumn{2}{c}{ Conductas orales } & \multicolumn{3}{c}{ Conductas locomotoras } \\
\hline Parámetros & O.R. & Valor de P & IC & O.R. & Valor de P & IC \\
\hline Contacto visual & 0,73 & 0,2488 & $0,42-1,24$ & 1,28 & 0,5214 & $0,60-2,73$ \\
Cama de paja & 1,43 & 0,1956 & $0,83-2,46$ & 1,2 & 0,5770 & $0,62-2,30$ \\
Tres raciones & 0,8 & 0,3103 & $0,52-1,22$ & 0,97 & 0,9069 & $0,60-1,55$ \\
Forraje $\geq 60 \%$ & 2,17 & 0,0001 & $1,45-3,22$ & 0,47 & 0,0098 & $0,27-0,83$ \\
Hembra & 1,38 & 0,1834 & $0,86-2,19$ & 1,63 & 0,0667 & $0,96-2,76$ \\
Macho entero & 1,49 & 0,1485 & $0,86-2,56$ & 1,06 & 0,8408 & $0,55-2,06$ \\
FSC & 0,58 & 0,0363 & $0,34-0,96$ & 1,45 & 0,2288 & $0,78-2,70$ \\
Caballo Chileno & 1,55 & 0,1386 & $0,86-2,19$ & 0,08 & 0,0190 & $0,55-2,06$ \\
\hline
\end{tabular}


presente estudio para una muestra que incluye, además de las razas FSC y Caballo Chileno, un tercer grupo de equinos mestizos utilizados para salto. Estas investigaciones dan cuenta de que en las poblaciones de equinos estudiadas existiría una frecuencia de presentación de conductas no deseadas menor a la reportada en otros países para equinos deportivos, lo que se puede deber a diferencias en la intensidad de los manejos realizados, o al tipo de actividad que realizan. A su vez esto determina que los factores de riesgo para la presentación de estas conductas, reportadas en otros países no serían necesariamente extrapolables a la realidad nacional.

En relación con la frecuencia de presentación de cada conducta, al igual que en la presente investigación, Pell y McGreevy (1999) y Waters y col (2002) también registraron la aerofagia con o sin fijación como la conducta no deseada estereotipada más frecuente, con un 5,7 y 10,5\% en cada estudio respectivamente, superada solo por la lignofagia con un $12 \%$ y 30,3\% (Pell y McGreevy 1999, Waters y col 2002). La lignofagia, conducta redirigida no estereotipada, también registró en este estudio una frecuencia de presentación alta (3\%) en comparación a las otras conductas (cuadro 2). De esta misma forma, Albright y col (2009) registraron una prevalencia en Estados Unidos de un 4,5\% de equinos con aerofagia, y Muñoz y col (2009) en su estudio con equinos Caballo Chileno reportaron una prevalencia de $2 \%$ para aerofagia semejantes a los resultados obtenidos en este trabajo. Tadich y col (2013) en el estudio realizado en equinos FSC en diferentes centros ecuestres de Chile encontraron prevalencias de 2,15\% y 2,02\% para aerofagia y lignofagia, respectivamente, y Tadich y col (2012) en su investigación de equinos Caballo Chileno, encontraron prevalencias de $2,5 \%$ y $8 \%$, respectivamente para aerofagia y lignofagia, siendo en ambos estudios las conductas no deseadas, estereotipada y no estereotipada, más frecuentes.

De esta forma, al igual que en otros países ambas conductas no deseadas son las que presentan la mayor frecuencia en equinos dedicados a distintas actividades deportivas, aunque en niveles inferiores a los reportados para el Reino Unido y Australia (Pells y McGreevy 1999, Waters y col 2002). En el caso del presente estudio puede que no se haya evidenciado una mayor proporción de lignofagia debido a que la mayoría de los ejemplares estudiados presentaron una frecuencia de alimentación de tres raciones al día, es así como McGreevy y col (1995) destacan que el uso de un menor número de raciones diarias podría facilitar la presentación de dicha conducta. Sin embargo, el análisis no permitió clasificar a esta variable como factor de riesgo. Un factor que explicaría la alta presentación de la conducta de aerofagia y lignofagia es que tienen una causa ambiental en común, como es el tipo de dieta alta en concentrados y baja en fibra que consumen estos animales, y que llevaría a un exceso de acidez gástrica (Hothersall y Nicol 2009), práctica alimentaria que se lleva a cabo en la gran mayoría de los centros ecuestres y que podría participar en el desarrollo de estas conductas no deseadas de tipo oral. Respecto de esto, Normando y col (2011) indican que los equinos con acceso a heno ad libitum estarían menos predispuestos a realizar la conducta de lignofagia que los que no lo poseen. Es importante destacar la relación entre la presentación de la conducta de lignofagia y aerofagia, a pesar de que la primera no se describe como estereotipia propiamente tal, y que simplemente podría reflejar una motivación por forrajear fuera de los horarios de alimentación debido a señales de saciedad, tiempo de ayuno, o baja en los niveles de glucosa sanguínea (Hothersall y Nicol 2009), es de relevancia, ya que se considera que esta conducta podría preceder o estar diretamente asociada al posterior desarrollo de la conducta de aerofagia (Nicol 1999, Waters y col 2002).

Hothersall y Nicol (2009) mencionan que la aerofagia es una conducta que no se observa en equinos domésticos asilvestrados o de vida libre, por lo que necesariamente las prácticas de manejo, en particular las relacionadas con la alimentación, causarían este comportamiento, ya que se asocia con la entrega de alimento concentrado, así como el balanceo estereotipado se asocia con la anticipación de la alimentación. Esto indicaría que las prácticas de manejo juegan un rol fundamental para la presentación de conductas no deseadas.

McGreevy y col (1995) sugieren como factor de riesgo una cantidad de forraje diario menor a $6,8 \mathrm{~kg}$, en el presente estudio el promedio de forraje entregado fue de $3 \mathrm{~kg}$, correspondiendo esta cantidad a menos de un $60 \%$ de la ración que reciben los animales, por lo que se esperaba que la regresión logística detectara el forraje $<60 \%$ como factor de riesgo. Si bien el porcentaje de forraje $\geq 60 \%$ se identificó como factor protectivo para las conductas de tipo locomotor (cuadro 4), lo cual concuerda con lo esperado, ya que se encuentra dentro del rango recomendado; en el caso de las conductas de expresión oral esta misma categoría de la variable ( $\geq 60 \%$ de forraje en la ración) resultó ser un factor de riesgo (cuadro 4). Este resultado es interesante, ya que concuerda con lo propuesto recientemente por McCall y col (2012) para la conducta oral estereotipada aerofagia, quienes proponen que el concentrado al ser un alimento altamente palatable y que en general se entrega en menor cantidad dentro de la ración, sería preferido por los equinos, siendo consumido primero y actuando como recompensa y refuerzo positivo para la conducta de aerofagia. En dicho estudio al entregar concentrado ad libitum, versus un grupo con cantidad controlada, reportaron una reducción en la frecuencia de presentación de la conducta (McCall y col 2012).

El balanceo estereotipado, conocido popularmente como mal del oso, fue una de conductas estereotipadas locomotoras de mayor presentación (cuadro 2), su frecuencia fue similar al 2,1\% de Pell y McGreevy (1997). Pero a nivel nacional Muñoz y col (2009) y Tadich y col (2012) registraron la conducta en una menor proporción con un $1 \%$ y $0,3 \%$ de su población respectivamente. Sin 
embargo, ambas investigaciones nacionales fueron acotadas a ejemplares de Caballo Chileno exclusivamente, a diferencia del resto de los estudios mencionados. Esta menor presentación de la conducta en los ejemplares de la raza Caballo Chileno concuerda con nuestros resultados, donde esta raza representa un factor protectivo para las conductas de origen locomotor (cuadro 4). La raza Caballo Chileno en general es mantenida por menos horas diarias en estabulación, factor de manejo que podría incidir en que la motivación de estos equinos por la locomoción y por interactuar con otros conespecíficos se vea satisfecha durante su manejo normal a pradera (Tadich y col 2012). En suma a esto, un estudio nacional, pero realizado con equinos FSC registró una prevalencia de 2,15\% para esta conducta estereotipada (Tadich y col 2013), semejante a la registrada en el presente trabajo, que incluyó individuos de raza FSC en mayor proporción, raza que tendría una mayor predisposición a la presentación de conductas no deseadas (Mills y col 2005). Sin embargo, no se identificó a la raza FSC como un factor de riesgo para la presentación de conductas no deseadas locomotoras como lo es el balanceo estereotipado.

En el estudio de Pell y McGreevy (1999) la caminata estereotipada se reportó en un 3,9\% de los equinos. Sin embargo, en el trabajo de Tadich y col (2013) y Tadich y col (2012) se registró una prevalencia de 2,02\% y 0,3\%, para FSC y Caballo Chileno, respectivamente. Aunque en el presente estudio se obtuvo una frecuencia de presentación menor a la reportada por Pell y McGreevy (1999), fue de todas maneras una de las conductas no deseadas estereotipadas más observada, y esta diferencia en su presentación probablemente se deba a que en aquel estudio se utilizaron exclusivamente equinos FSC, mientras que en la presente investigación se incluyeron también equinos mestizos y Caballo Chileno, encontrándose 14 ejemplares FSC y 16 equinos mestizos que presentaron esta conducta, mientras que solo se presentó en uno de los ejemplares Caballo Chileno, lo que explicaría la menor presentación en el total de la muestra en este trabajo y en el de Tadich y col (2012). Esto concuerda con el resultado que indica a la raza Caballo Chileno como factor protectivo para el desarrollo de conductas locomotoras en el presente estudio, pero se contradice con el estudio de Muñoz y col (2009), quienes la reportaron como la conducta no deseada más frecuente, sin embargo, hay que tomar en consideración que dichos autores seleccionaron una muestra de ejemplares más pequeña $(n=100)$ de equinos Caballo Chileno.

La ausencia de contacto social ha sido reportada como un importante factor de riesgo para la presentación de conductas no deseadas (McGreevy y col 1995). Esto tiene sentido si recordamos que los equinos son animales gregarios, para los cuales la aislación social representa una experiencia altamente perturbadora (Mal y col 1991). Ambos tipos de conducta (oral y locomotora) podrían ser dependientes del contacto visual entre conespecíficos, es así como Mills y Riezebos (2005) concluyeron que la conducta de balanceo estereotipado y cabeceo, ambas conductas locomotoras, fueron significativamente menores en los equinos a los que se les proporcionó una imagen de otro equino dentro de la pesebrera, el mismo efecto se ha visto incorporando ventanas en las pesebreras (Cooper y col 2000).

Si bien el sexo no resultó ser un factor de riesgo estadísticamente significativo (cuadro 4), sí se observó una asociación cercana a significativa para el sexo hembra (yeguas). Existen variados resultados en la literatura respecto del efecto del sexo sobre el desarrollo de estas conductas, mientras algunos estudios registran una asociación significativa entre el sexo hembra y la presentación de estereotipias en equinos FSC (Tadich y col 2013); otros determinaron una mayor prevalencia en hembras y machos castrados (Mills y col 2002). A diferencia de la presente investigación, ellos sí encontraron una diferencia significativa en la prevalencia de estereotipias entre los sexos. Sin embargo, estos investigadores evaluaron exclusivamente equinos FSC, mientras que en el presente trabajo al evaluar tres tipos de razas pudo llevar a una conclusión diferente. En cuanto a los ejemplares de raza Caballo Chileno, Tadich y col (2012) registraron una asociación significativa entre las conductas anormales y los machos enteros, mientras que Muñoz y col (2009) observaron una diferencia significativa entre machos enteros y hembras solo en la conducta de caminata estereotipada, siendo más frecuente en los machos enteros, por lo que probablemente los equinos Caballo Chileno de este sexo tengan mayor predisposición a la presentación de esta conducta, debido al manejo más intensivo en cuanto a estabulación y actividad (deportiva y reproducción) al que son sometidos en relación con las hembras y machos castrados de la misma raza.

La raza Caballo Chileno, cuya actividad es el rodeo, resultó ser un factor protectivo frente a las conductas no deseadas de origen locomotor (cuadro 4), pero en este caso la variable tipo de raza involucraría la genética, los manejos a los que son sometidos y el tipo de actividad que realizan, ya que cada raza consultada realizaba una actividad en particular. En cuanto a la actividad, Normando y col (2011) mencionan que el estilo de monta Inglés, que se asemeja a lo experimentado por los equinos destinados a carrera y salto, tendría más probabilidades de sufrir estereotipias locomotoras u orales, así como otros problemas conductuales, además tienden a permanecer bajo una estabulación más restrictiva que los equinos con otros estilos de equitación, como el estilo Occidental, similar al de los equinos de rodeo, que al permanecer menos tiempo en estabulación tienen menos probabilidades de presentar estereotipias.

Respecto de la conducta de la raza Caballo Chileno hay escasa información disponible, pero posiblemente este tipo de raza sea un factor protectivo al compararla con las otras razas debido a que se caracteriza por ser un sistema menos intensivo o por una menor predisposición propia de la raza en comparación a las demás, lo que concuerda con lo encontrado por Christie y col (2006), 
quienes concluyeron que los equinos de tiro y miniatura, al igual que lo encontrado para los equinos de tipo Caballo Chileno, presentan menos riesgo de presentar estereotipias orales. Además, se describe en la literatura que la raza FSC estaría más predispuesta a presentar conductas no deseadas, como lo señala el estudio de Albright y col (2009) quienes reportaron una predisposición racial para la presentación de la conducta oral de aerofagia con fijación, en dicho estudio la prevalencia de aerofagia alcanzó un 13,3\% para el FSC, mientras que los equinos tipo poni, como el Caballo Chileno, solo alcanzaron una prevalencia de $1,7 \%$. En suma a esto, McGreevy (2004) también reporta que los FSC están más predispuestos que otras razas a realizar aerofagia con fijación y balanceo estereotipado, y Luescher y col (1998) encontraron una prevalencia de $2,99 \%$ de aerofagia en ponis, en contraste con el 6,8\% en los FSC, a su vez no se registraron ponis que presentaran la conducta de patear la pesebrera, balanceo y caminata estereotipada. En el caso de este estudio la raza FSC se presentó como factor protectivo para la presentación de conductas orales (cuadro 4). Sin embargo, la presencia de variables confundentes, como grupo etario, regiones y sexo (Rial y Varela 2008) podría explicar este resultado. La raza Caballo Chileno se caracteriza por ser un animal rústico y de temperamento estable, la relación entre temperamento y riesgo de desarrollar estas conductas sería interesante de estudiar en mayor profundidad en investigaciones posteriores.

Finalmente, llama la atención que el uso de viruta como material de cama no resultara ser un factor de riesgo para la presentación de conductas no deseadas, ya que investigadores como McGreevy y col (1995) mencionan que un material de cama diferente a la paja aumenta el riesgo de presentar conductas anormales. Werhahn y col (2010), al medir el tiempo de ocupación de la cama, concluyeron que la paja es el material de cama preferido por los equinos al compararlo con otros materiales como la viruta. Sin embargo, no se puede descartar que el resultado obtenido se vea influenciado por la falta de homogeneidad de la muestra, ya que no existieron proporciones semejantes entre los dos materiales de cama, llegando a un $88 \%$ los individuos que poseían camas de viruta (cuadro 3), y por otra parte la calidad y el manejo del material utilizado, como la mantención de la higiene (niveles de amonio y humedad) así como la profundidad de la cama (McGreevy 2004) características que podrían tener un rol importante al momento de considerarse como factor protectivo o de riesgo.

El presente estudio aporta datos importantes, presentando evidencia de que la realización de ciertas prácticas de manejo y características del animal pueden favorecer el desarrollo de alteraciones conductuales. Así también se condice con estudios recientes que reportan que bajas proporciones de concentrado en la dieta también podrían jugar un rol importante en el desarrollo de conductas de tipo oral como la aerofagia. Es fundamental que los propietarios tomen estos factores en cuenta al momento de diseñar sus sistemas de mantención de equinos para así poder resguardar su bienestar.

\section{REFERENCIAS}

Albright J, H Mohammed, C Heleski, C Wickens, K Houpt. 2009. Cribbiting in US horses: breed predispositions and owner perceptions of aetiology. Equine Vet $J$ 41, 455-458.

Boyd LE. 1986. Behavior problems of equids in zoos. Vet Clin N AmEquine Pract 2, 653-664.

Christie J, C Hewson, C Riley, M McNiven, I Dohoo, L Bate. 2006. Management factors affecting stereotypies and body condition score in nonracing horses in Prince Edward Island. Can Vet J 47, 136-143.

Cooper JJ, L McDonald, DS Mills. 2000. The effect of increasing visual horizons on stereotypic weaving: implications for the social housing of stabled horses. Appl Anim Behav Sci 69, 67-83.

Hosmer D, S. Lemeshow 2000. Applied logistic regression. $2^{\text {nd }}$ ed. Wiliey-Interscience, Victoria, Canada.

Hothersall B, C Nicol. 2009. Role of diet and feeding in normal and stereotypic behaviors in horses. Vet Clin North Am Equine Pract $25,167-181$

Hothersall B, R Casey. 2012. Undesired behaviour in horses: a review of their development, prevention, management and association with welfare. Equine Vet Educ 24, 479-485.

Houpt KA. 1986. Stable vices and trailer problems. Vet Clin N AmEquine Pract 2, 623-644.

Luescher U, D McKeown, H Deant. 1998. A cross-sectional study on compulsive behaviour (stable vices) in horses. Equine Vet $J 30$ Suppl 27, 14-18.

Mal ME, TH Friend, DC Lay, SG Vogelsang, OC Jenkins. 1991. Physiological responses of mares to short-term confinement and isolation. J Equine Vet Sci 11, 96-102.

Mason G. 1991. Stereotypies: a critical review. Anim Behav 41, 1015-1037.

McCall CA, TR Fenn, WH McElhenney, WH Brown, PJ Tyler. 2012. Cribbing behavior of horses consuming ad libitum concentrate feed. PAS 28, 403-409.

McGreevy P, PJ Cripps, NP French, LE Green, CJ Nicol. 1995. Management factors associated with stereotypic and redirected behaviour in the thoroughbred horse. Equine Vet J 27, 86-91.

McGreevy P. 1997. Do stabled horses cope? J Biol Educ 31, 207-211.

McGreevy P. 2004. Equine behavior: a guide for veterinarians and equine scientists. Saunders, Elsevier, London, UK.

Mills D, R Alston, V Rogers, N Longford. 2002. Factors associated with the prevalence of stereotypic behaviour amongst Thoroughbred horses passing through auctioneer sales. Appl Anim Behav Sci 78, 115-124.

Mills D, M Riezebos. 2005. The role of the image of a conspecific in the regulation of stereotypic head movements in the horse. Appl Anim Behav Sci 91, 155-165.

Mills D, K Taylor, J Cooper. 2005. Weaving, headshaking, cribbing, and other stereotypies. AAEP Proceedings 51, 1-5.

Muñoz L, J Torres, O Sepúlveda, C Rehhof, R Ortiz. 2009. Frecuencia de comportamientos anormales estereotipados en caballos criollo chileno estabulados. Arch Med Vet 41, 73-76.

Nicol C. 1999. Understanding equine stereotypies. Equine Vet J Suppl $28,20-25$.

Normando S, L Meers, W Ellery, M Faustini, F Ödberg. 2011. Variables affecting the prevalence of behavioural problems in horses. Can riding style and other management factors be significant? Appl Anim Behav Sci 133, 186-198.

NRC, National Research Council. 1989. Nutrient requirements of horses. Subcommittee on Horse Nutrition, National Academies, Washington D.C., USA.

Pell S, P McGreevy. 1999. Prevalence of stereotypic and other problem behaviours in thoroughbred horses. Aust Vet J 77, 678-679.

Rial A, J Varela. 2008. Estadística aplicada a la epidemiología y al diagnóstico. En: Rial A, Varela J (eds). Estadística práctica para 
la investigación en ciencias de la salud. Netbiblo, La Coruña, España, Pp 299-319.

Stephens N. 2011. Descripción de conductas anormales, estereotipadas y no estereotipadas, en equinos Fina Sangre de Carrera (FSC) y razas de equitación destinados a competencias en Chile. Memoria de título, Escuela de Ciencias Veterinarias, Universidad de Viña del Mar, Viña del Mar, Chile.

Tadich T, O Araya. 2010. Conductas no deseadas en equinos. Arch Med Vet 42, 29-41.

Tadich T. 2012. Destete y entrenamiento: períodos críticos para el bienestar del caballo criollo chileno. Tesis Doctoral, Facultad de Ciencias Veterinarias, Universidad Austral de Chile, Valdivia, Chile.

Tadich T, JP Smulders, O Araya, C Nicol. 2012. Husbandry practices associated with the presentation of abnormal behaviours in chilean creole horses. Arch Med Vet 44, 279-284.
Tadich T, C Weber, CJ Nicol. 2013. Prevalence and factors associated with abnormal behaviors in chilean racehorses: a direct observational study. J Equine Vet Sci 33, 95-100.

Waters AJ, CJ Nicol, NP French. 2002. Factors influencing the development of stereotypic and redirected behaviours in young horses: findings of a four year prospective epidemiological study. Equine Vet J 34, 572-579.

Weber C. 2010. Prevalencia y descripción de conductas estereotipadas en equinos purasangre inglés destinados a carrera en Chile. Memoria de título, Escuela de Medicina Veterinaria, Universidad Austral de Chile, Valdivia, Chile.

Werhahn H, E Hessel, I Bachhausen, H Van Den Weghe. 2010. Effects of different bedding materials on the behavior of horses housed in single stalls. J Equine Vet Sci 30, 425-431. 